\title{
Calidad y tiempo de llenado de las anotaciones de enfermería en un Servicio de Emergencia de Lima - 2018.
}

Quality and time of filling nursing annotations in the emergency service, Lima - 2018.

\author{
Lourdes Alvarez Antezana ${ }^{1, a}$.
}

RESUMEN

Objetivo: Determinar la calidad de las anotaciones de enfermería y el tiempo de llenado en el Servicio de Emergencia durante el segundo semestre del 2018. Material y métodos: Estudio descriptivo de corte transversal. Se trabajó con la totalidad de la población de enfermeras que laboran en el servicio que cumplían los criterios de inclusión, la muestra de anotaciones fue 245. Para la recolección de datos se realizó la técnica de análisis documental, se elaboraron dos instrumentos, el primero evaluó: criterios de legalidad - estructura y contenido, el segundo fue una lista de cotejo, donde se registró el tiempo en minutos. Resultados: De las 245 anotaciones de enfermería evaluadas, el $100 \%$ presentó mala calidad. Según la evaluación del criterio de legalidad en las anotaciones, el 19,6\% tiene buena calidad, donde solo el 2,9\% obtuvo el puntaje máximo: 17 , mientras el 16,7\% obtuvo como puntaje 16 . Se obtuvo una media de 6,4 minutos DS=3,0 teniendo como rango inferior 3,4 y rango máximo 9,4 minutos de tiempo para la elaboración de las anotaciones de enfermería. Conclusiones: Las anotaciones de enfermería revelan mala calidad y el tiempo utilizado por cada enfermera con 10 pacientes a su cargo demora entre 34 minutos a 1 hora con 34 minutos. Los resultados justifican la implementación de una anotación de enfermería que cumpla criterios con mayor énfasis en relación a criterios de contenido, que cuente con la nomenclatura NANDA, NIC, NOC.

PALABRAS CLAVE: Registros de enfermería, control de calidad, tiempo.

\section{SUMMARY}

Objective: To determine the quality of the note nursing and the filling time in the emergency service during the second trimester of 2018. Material and methods: Descriptive study of transverse cut, we worked with the whole population of nurse who works in the service and meets the inclusion criteria, the sample of notes was 245 . For the data recollection, it was performed the techniques of documentary analysis, two instruments were developed, the first one evaluated: legality criteria - structure and content, the second one was a checklist, where time was recorded in minutes. Results: Of the 245 notes nursing evaluated, $100 \%$ have poor quality. According to the evaluation of the criterion of legality in the notes, $19.6 \%$ have good quality, where only $2.9 \%$ got the maximum score of 17 . Whereas, the $16.2 \%$ got a score 16 . An average of 6.4 minutes was obtained with a standard deviation of 3.0, with a lower range of 3.4 and a maximum range of 9.4 minutes for the preparation of nursing notes. Conclusions: The quality of the total nursing notes evaluated was poor. The time used by each nurse with 10 patients under their care takes between 34 minutes to 1 hour and 34 minutes. The results justify the implementation of a nursing note that meets criteria with greater emphasis in relation to content criteria, which has the nomenclature NANDA, NIC, NOC.

KEY WORDS: Nursing records, quality control, time.

Hospital Cayetano Heredia. Lima, Perú.

Licenciada Especialista en Unidad de Cuidados Intensivos. 


\section{INTRODUCCIÓN}

El registro de enfermería es la herramienta a través de la cual se evalúa el nivel de la calidad técnico científica, humana, ética y la responsabilidad del profesional de enfermería. Los registros son una documentación donde se recoge toda la información relativa al proceso asistencial, girando en torno al paciente y sus necesidades (1). Estos son formatos obligatorios de la historia clínica del paciente que tienen carácter jurídico - legal, los cuales son: anotaciones de enfermería, Kardex, Balance Hídrico, Hoja gráfica. Así, las anotaciones de enfermería son registros de gran relevancia en el quehacer del profesional en su ejercicio asistencial, en docencia e investigación (2).

Al respecto, en el artículo de Nuñez, menciona que el Ministerio de Salud, estableció que el tipo de anotaciones de enfermería a utilizarse en los establecimientos de Salud debe contemplar datos Subjetivos, Objetivos, Análisis, Planificación, Intervención y Evaluación - SOAPIE (1).

Entre los registros mencionados; las anotaciones de Enfermería; contienen información sobre el progreso del paciente, sin embargo se puede observar que existe diversidad de anotaciones, que cada institución adecuó a su realidad (3).

En la actualidad los profesionales de enfermería no asumen la elaboración de los registros como parte del área asistencial sino administrativa, lo que les resta tiempo para la atención del paciente y sus labores asistenciales, muchas veces incumpliendo el adecuado registro, faltando a la responsabilidad ético-moral, respeto a los pacientes y responsabilidad profesional; acortando así la continuidad de los cuidados de calidad, ya que su labor se realiza por turnos y es secuencial. Otras razones por parte del personal que justifican su incumplimiento son la carga de trabajo asistencial, dificultades de la metodología (taxonomía NANDA, NIC y NOC), desactualización de guías de intervención, inadecuados formatos que se aplican, inadecuada infraestructura y falta de espacios (2).

En América latina, estudios en Cuba y Brasil encontraron deficiencias en las anotaciones de enfermería como la ausencia de diagnósticos de enfermería, ausencia de las notas en las historias clínicas y la demanda de tiempo para la aplicación, la cual fluctúa entre 35 y 140 minutos (4).

En Perú se han realizado estudios en diferentes hospitales, como el Instituto de Salud del Niño,
Hospital Daniel Alcides Carrión, en un hospital del MINSA y otro de ESSALUD en Chachapoyas, donde se encontró la misma problemática: ausencia de diagnósticos de enfermería, notas de enfermería incompleta, así como los factores personales e institucionales de las enfermeras que justifican su incumplimiento $(5,6,7)$.

En el servicio de Emergencia del Hospital Nacional Cayetano Heredia $(\mathrm{HNCH})$ se cuenta con un registro de notas de enfermería donde se consigna datos según la Norma técnica de salud para la gestión de la historia clínica: NT $\mathrm{N}^{0}$ 022-MINSA/DGSP-V02(8). Para evaluar su cumplimiento, se realizan auditorias por parte del área de calidad de la institución, estas se rigen en base a la norma técnica y a una guía elaborada por las enfermeras del servicio en el 2012, donde los diagnósticos se encuentran sin la taxonomía NANDA, se consignan objetivos, las intervenciones de enfermería sin taxonomía NIC y resultados sin considerar NOC; habiendo encontrado deficiencias en el llenado. Si la situación mencionada continúa, los resultados de la evaluación de calidad no reflejarán el verdadero actuar de cada uno de los profesionales de enfermería, ya que las anotaciones son los que evidencian la aplicación del Proceso de Atención de Enfermería (PAE) en el cuidado del paciente y por ende permiten la seguridad y la evolución del paciente, favoreciendo el cuidado individualizado. El objetivo del presente trabajo fue determinar la calidad de las anotaciones de enfermería y el tiempo de llenado en el servicio de Emergencia del HNCH durante el segundo semestre del 2018, constituyendo uno de los pocos trabajos de investigación que evalúa la calidad de las anotaciones sobre contenido (NANDA - NIC - NOC), criterios de legalidad y el tiempo en la elaboración de las mismas.

\section{MATERIAL Y MÉTODOS}

El presente estudio es de tipo descriptivo de corte transversal, ya que los datos recolectados en un periodo de tiempo se detallan sin intervención del investigador, respetando las consideraciones éticas de autonomía, justicia, beneficencia y no maleficencia. La población estuvo conformada por las anotaciones de enfermería registradas en las historias clínicas de pacientes hospitalizados solo en los tópicos de observación y medicina del servicio de Emergencia del $\mathrm{HNCH}$, llegando a un total de 94620 pacientes en el año 2017. Así mismo se trabajó con la población de enfermeras del servicio, siento un total de 92 enfermeras entre nombrados, contratados y contrato 
por terceros del Servicio de Emergencia Adultos. La investigación fue aprobada y autorizada por el Comité de Ética del Hospital Nacional Cayetano Heredia.

La muestra se obtuvo mediante la aplicación de la fórmula proporcional y comprendió a 245 anotaciones de enfermería de historias clínicas de pacientes hospitalizados en el servicio de emergencia, tomando en cuenta los siguientes criterios de inclusión: Ser anotaciones de enfermería del servicio de emergencia llenado por la enfermera asistencial; ser anotaciones de enfermería elaborados durante la aplicación de estudio; anotaciones de enfermería de los tópicos de medicina y observación. Enfermeras asistenciales que deseen participar en el estudio. Criterios de Exclusión: Anotaciones de enfermería de pacientes de alta o fallecidas. Enfermeras asistenciales que se encuentren de vacaciones, licencias.

La técnica que se utilizó fue el análisis documental, describiendo la anotación de enfermería según su estructura externa e interna, para lo cual se elaboraron dos instrumentos. El primer instrumento constó de dos dimensiones de valoración de las anotaciones de enfermería, sobre: criterios de legalidad (cumpliendo la Norma técnica de salud para la gestión de la historia clínica: NT No 022-MINSA/DGSP-V02), criterios de estructura y contenido, haciendo referencia del uso adecuado del cumplimiento del proceso de atención de enfermería a través del SOAPIE (Datos Subjetivos, Objetivos, Análisis, Planificación, Intervención, Evaluación), en base a la nomenclatura universal de NANDA - NIC - NOC, y la relación y coherencia entre cada etapa del SOAPIE. El segundo instrumento fue una lista de cotejo, en el cual se registró el tiempo en minutos de realización de las anotaciones, según etapa culminada durante el turno evaluado.

Los instrumentos elaborados se sometieron a validez de contenido, aplicando la V de AIKEN, cuantificando la relevancia de los ítems del instrumento respecto al dominio de contenido a partir de la valoración del juicio de los expertos, contando con 5 profesionales de enfermería con experiencia en el tema propuesto. $\mathrm{Al}$ obtener validez aceptable y validez fuerte con un puntaje entre 0,81 a 1,0 según la interpretación de resultados.

Luego se procedió a realizar las coordinaciones administrativas correspondientes para luego iniciar las evaluaciones con las enfermeras y anotaciones realizadas en el servicio de emergencia en el periodo programado. Los datos fueron codificados y procesados a través del programa SPSS versión 23.0 y luego se obtuvieron las medidas estadísticas descriptivas, como frecuencias absolutas y relativas, además de medidas de tendencia central para las variables requeridas.

\section{RESULTADOS}

Fueron evaluadas 245 anotaciones de enfermería elaborados por los profesionales de enfermería del servicio de emergencia. El total de anotaciones tiene una mala calidad.

La evaluación de la calidad se realizó a través del cumplimiento de dos dimensiones: criterio de legalidad y de estructura y contenido, de los que se encontraron los siguientes resultados: Del total de anotaciones de enfermería analizadas el 19,6\% (48), presenta buena calidad según criterios de legalidad (tabla 1). El 16,7\% tiene puntaje de 16 y el 2,9\% puntaje máximo (tabla 2).

De las anotaciones que tienen una buena calidad en el criterio de legalidad el 8,3\% no registró la hora, el $14,6 \%$ no registró la evaluación de las intervenciones de enfermería y el $62,5 \%$ no redactó con letra clara y legible los datos del plan de cuidados (tabla 3). Según el criterio de Estructura y Contenido el $100 \%$ de las anotaciones tiene una mala calidad.

En la evaluación de tiempo de llenado se obtuvo una media de 6,50 y DS 3,01 minutos, teniendo como rango inferior 3,4 minutos y rango máximo de 9,4 minutos de tiempo para la elaboración de las anotaciones de enfermería (tabla 4).

Tabla 1. Calidad de las anotaciones de enfermería según criterio de legalidad

\begin{tabular}{cccc}
\hline & Calidad & Frecuencia & \% \\
\hline \multirow{3}{*}{ Válido } & Mala & 197 & 80,4 \\
& Buena & 48 & 19,6 \\
& Total & 245 & 100,0 \\
\hline
\end{tabular}

Tabla 2. Puntaje de la evaluación según criterio de legalidad

\begin{tabular}{cccc}
\hline & Puntaje & Frecuencia & $\mathbf{\%}$ \\
\hline \multirow{3}{*}{ Válido } & $8-12$ & 11 & 4,5 \\
& $13-16$ & 227 & 92,6 \\
& 17 & 7 & 2,9 \\
& Total & 245 & 100,0 \\
\hline
\end{tabular}


Tabla 3. Ítems obviados del criterio de legalidad para obtener una buena calidad de las anotaciones

\begin{tabular}{|c|c|c|c|c|c|c|c|}
\hline & & \multicolumn{6}{|c|}{ Criterio de Legalidad } \\
\hline & & \multicolumn{2}{|c|}{ Mala } & \multicolumn{2}{|c|}{ Buena } & \multicolumn{2}{|c|}{ Total } \\
\hline & & Recuento & $\%$ & Recuento & $\%$ & Recuento & $\%$ \\
\hline \multirow{2}{*}{$\begin{array}{l}\text { Se registra hora de las } \\
\text { intervenciones }\end{array}$} & No & 106 & 53,8 & 4 & 8,3 & 110 & 44,9 \\
\hline & $\mathrm{Si}$ & 91 & 46,2 & 44 & 91,7 & 135 & 55,1 \\
\hline \multirow{2}{*}{$\begin{array}{l}\text { Se registra Evaluación de las } \\
\text { intervenciones }\end{array}$} & No & 153 & 77,7 & 7 & 14,6 & 160 & 65,3 \\
\hline & $\mathrm{Si}$ & 44 & 22,3 & 41 & 85,4 & 85 & 34,7 \\
\hline $\begin{array}{l}\text { Los datos del plan de cuidados } \\
\text { es redactada con letra clara y }\end{array}$ & No & 186 & 94,4 & 30 & 62,5 & 216 & 88,2 \\
\hline legible y sin enmendaduras & $\mathrm{Si}$ & 11 & 5,6 & 18 & 37,5 & 29 & 11,8 \\
\hline
\end{tabular}

Tabla 4. Tiempo de elaboración de las anotaciones de enfermería

\begin{tabular}{lccccc}
\hline & N & Mínimo & Máximo & Media & Desviación estándar \\
\hline $\begin{array}{l}\text { Anotaciones de } \\
\text { Enfermería }\end{array}$ & 245 & 2,00 & 17,00 & 6,49 & 3,05 \\
N válido (por lista) & 245 & & & & \\
\hline
\end{tabular}

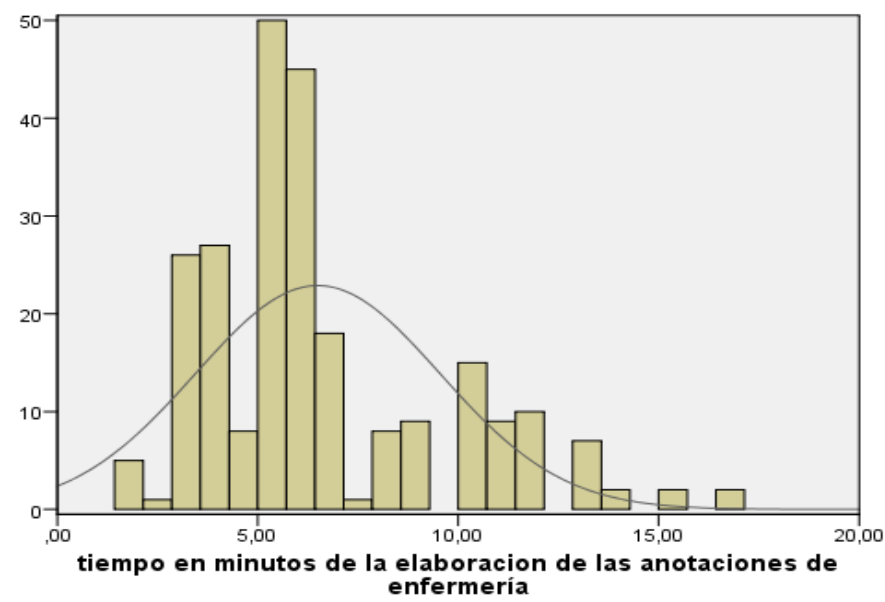

Grafico 1. Distribución del tiempo en minutos de la elaboración de las anotaciones de enfermería

Para el tiempo de elaboración de las notas de enfermería, el tiempo que más se utilizó fue de 5 minutos $(17,1 \%)$, teniendo como valor mínimo 2 minutos $(2 \%)$ y un valor máximo de 17 minutos $(0,8 \%)$ (gráfico 1$)$.

\section{DISCUSIÓN}

El objetivo de la presente investigación fue determinar la calidad de las anotaciones de enfermería y el tiempo de llenado. Diversos trabajos de investigación a nivel internacional han encontrado inconsistencias en la elaboración de las anotaciones de enfermería y la calidad de las mismas (9) en nuestro medio también se han realizado diversos trabajos en diferentes hospitales a nivel nacional destacando los elaborados por Grespan V., Contreras P., Fretel V. y Valverde A $(5,6,10,11)$.

En el presente estudio se evidenció que el 100\% obtuvo mala calidad. Esto corrobora los resultados encontrados por Borsato G, Pokorski S., Silva J., Grespan S., Méndez C., y Valverde A. (11-14), quienes mostraron que existen varias deficiencias en la aplicación del proceso de atención de enfermería, disminuyendo su calidad. En el estudio de Bartolo E. 
refirieron que los factores institucionales desfavorecen en la elaboración de las anotaciones de enfermería, evidenciando la mala y regular calidad (15). Un estudio realizado en Brasil demostró que ninguno de los ítems bajo evaluación alcanzó el criterio de buen estándar de calidad en su llenado (16); mientras que tro estudio en Perú - Chachapoyas solo el 36,6\% mostraron un llenado regular (7).

Haciendo un análisis individual de las dimensiones de calidad, se encuentra que en el criterio de legalidad, un porcentaje elevado tiene mala calidad, mientras que un menor porcentaje son buenas, de estas, 41 anotaciones tuvieron por lo menos una omisión de registro de hora, registro de evaluación o claridad de la letra, solo 7 de ellas tuvieron el puntaje máximo. Esto corrobora los datos encontrados por Grespan, donde encontró defectos respecto al lenguaje, gramática y claridad (4). En el estudio realizado por Mejía D. se encontró que solo el 33,1\% registro la hora de las intervenciones (17). Otro estudio, en relación a la evaluación de las intervenciones encontró que solo el $16 \%$ realiza la evaluación de los cuidados brindados durante el trabajo (10).

En el criterio de estructura y contenido, se hace referencia al uso adecuado del cumplimiento del proceso de atención de enfermería a través del SOAPIE, en base a la nomenclatura universal de NANDA - NIC - NOC, y la relación y coherencia entre cada etapa del SOAPIE, en el estudio se encuentra que el total de anotaciones evaluadas tienen mala calidad. Estos resultados difieren con los encontrados en los estudios de Grespan donde el $26.7 \%$ tiene mala calidad y el $64,6 \%$ tiene regular calidad, además evidencian que el $59 \%$ de los registros no contaban con diagnósticos de enfermería (4), en el estudio de Méndez encontraron que el $80,6 \%$ del personal desconoce las normas elaboradas para los registros de enfermería (14), en un estudio en otro departamento del Perú, se evidencia que la capacidad científico - técnica de las anotaciones de enfermería es poca en ambas instituciones en Chachapoyas MINSA-ESSALUD, pero la realización de las mismas se cumple en las historias clínicas (7).

Dos estudios, llegaron a la conclusión que más de la mitad de su población de estudio no consideran entre sus criterios de elaboración datos importantes en base al PAE $(10,18)$. Otro estudio en relación al SOAPIE, encontró que la calidad de los registros fue de medio a bajo a pesar que estuvieron en base a la estructura del SOAPIE, pero en estos no registran datos subjetivos, plan de intervención ni análisis (11).
Estos resultados corroboran con nuestros resultados en relación a los 9 criterios de estructura y contenido de nuestra evaluación.

La presente investigación muestra una media de tiempo de 6,49 minutos para la elaboración de una anotación de enfermería. Al realizar un cálculo matemático con el número de pacientes a cargo (10 pacientes) de la enfermera de emergencia en un turno de 12 horas, resulta que la enfermera utiliza entre 64 +/- 30 minutos, lo que equivale a un rango de tiempo de 34 minutos a 1 hora con 34 minutos, en un solo formato de enfermería, pero en el ejercicio diario la enfermera por lo menos debe llenar los siguientes formatos (Kardex, Balance hídrico, Hoja gráfica, Hoja de procedimientos, censo de paciente, indicadores, cuaderno de reporte), haciendo una sumatoria aproximada de los minutos utilizados, con la totalidad de los registros, se puede evidenciar el uso de por lo menos 5 horas en llenado de formatos.

Estos datos son corroborados por el estudio de Avendaño y colaboradores, donde la media resultó en $26+/-10$ minutos en el formato estandarizado propuesto. Gonzales e Ibarra, citado por Avendaño, mencionan que las anotaciones de enfermería son variadas, complejas y requieren tiempo pues las enfermeras pasan entre 35 y 140 minutos escribiendo por turno (2). En otro estudio, entrevistaron a 48 enfermeros, el 64,6\% respondió que no aplican el PAE por falta de tiempo y la cantidad de pacientes (19). En el estudio de contreras aplicaron un cuestionario donde el 63\% refiere que el tiempo es importante, llegando a la conclusión que el tiempo laboral que tienen para la realización de las anotaciones es mínimo debido a la recargada labor asistencial.

\section{CONCLUSIONES}

Con estos resultados se concluye que el tiempo que utiliza cada enfermera en la elaboración de documentos administrativos es de 34 minutos a 1 hora con 34 minutos, a pesar del tiempo utilizado, la calidad de las anotaciones es mala, según la evaluación del criterio de estructura y contenido, así como un bajo porcentaje de la evaluación del criterio de legalidad donde solo un 19,6\% presentó buena calidad. Por lo encontrado en esta investigación, estos resultados justifican la implementación de una anotación de enfermería estructurada que cumpla los criterios de legalidad, de estructura y contenido, con mayor énfasis en relación a criterios de contenido, ya que al contar con la nomenclatura NANDA - NIC - NOC, se 
podría mejorar la calidad de las anotaciones y reducir el tiempo para la elaboración de documentación administrativa, incrementando el tiempo para el cuidado directo en la relación enfermero(a)- paciente.

\section{Correspondencia:}

Lourdes Alvarez Antezana.

Correo electrónico: lulu19.lourdes@gmail.com

\section{REFERENCIAS BIBLIOGRÁFICAS}

1. Nuñez A, Rodriguez L, Díaz R, Cervera $M$, Constantino F. Incorporando las anotaciones de enfermería tipo SOAPIE en el servicio de Pediatría. Rev ACC CIETNA (Per). 2018; 5 (2): 66 - 73.

2. Avendaño G, Ramírez T, Colin L, Rentería C, López C. Exactitud, precisión y curva de aprendizaje del instrumento para los registros clínicos de enfermería. Estudio comparativo. Rev Esp Med Quir (Esp). 2011; 16 (2): $97-103$.

3. Gutiérrez O. Factores que determinan la aplicación del proceso de enfermería en instituciones hospitalarias de Villavicencio, Colombia. Rev Av.enferm (Col). 2009; 27(1): 60 - 68 .

4. Grespan V, Innocenzo M. Evaluación de la calidad de los registros de enfermería en la historia clínica por medio de la auditoria. Acta Paul Enferm (Bra). 2009; 22 (3) 313- 317.

5. Asencios V. Efectividad de una guía de diagnósticos de enfermería para el registro en pacientes con cardiopatías. Tesis de licenciatura. Lima, Perú: Universidad Nacional Mayor de San Marcos; 2014.

6. Contreras P. Factores personales e institucionales que influyen en el llenado de notas de enfermería del servicio de emergencia - Hospital Nacional Daniel Alcides Carrión. Rev Perú Obstet Enferm 2012; 8 (2): $93-98$.

7. Rodríguez M. Manejo de Herramientas de gestión y percepción del cuidado de enfermería en hospitales de Chachapoyas Perú. Enferm univ. 2014; 11(1): 3-10.

8. Ministerio de Salud. Dirección General de Salud de las Personas. Norma técnica de salud para la gestión de la historia clínica: NT No 022-MINSA/DGSP-V02. Lima : Ministerio de Salud. (Citado en junio del 2019) Disponible en: http://bvs.minsa.gob.pe/local/ dgsp/NT022hist.pdf

9. Fernández S, Ruy Díaz K, Del Toro M. Notas de Enfermería: Una mirada a su calidad. Rev. Salud
Uninorte. Barranquilla (Col). 2016; 32(2): 337-345.

10. Fretel V. Evaluación de las anotaciones de enfermería durante los años 2000 - 2002 en el servicio de Neurología del hospital Nacional Guillermo Almenara Irigoyen. Tesis de licenciatura. Lima, Perú: Universidad Nacional Mayor de San Marcos; 2004.

11. Valverde A. Calidad de los registros de enfermería elaborados por las enfermeras del servicio del centro quirúrgico del instituto nacional de salud del niño, 2009. Tesis de Especialista en Enfermería. Lima, Perú: Universidad Nacional Mayor de San Marcos; 2013.

12. Borsato G, Rossaneis A, Fernández $H$, Oliveira M, Willamowius D. Evaluación de la calidad de las anotaciones de enfermería en un hospital universitario. Acta Paul Enferm (Bra). 2011; 24(4):527 - 533.

13. Pokorski S, Morales A, Chiarelli R, Paganin A, Rejane E. Proceso de enfermería: de la literatura a la práctica: ¿Qué estamos haciendo, de hecho? Rev. Latino - am. Enfermagem (Bra). 2009; 17(3): 0-0.

14. Méndez G, Morales M, Preciado M, Ramírez M, Sosa V, Jiménez M. La trascendencia que tienen los registros de enfermería en el expediente clínico. Rev. Mexicana de Enfermería Cardiológica. 2001; 9 (4): $11-17$.

15. Bartolo C, Solórzano M. Factores personales e institucionales relacionados a la calidad de las anotaciones de enfermería en el servicio de cirugía del Hospital Guillermo Almenara Irigoyen, abril junio, 2011. Rev. Científica de Ciencias de la Salud (Per). 2011; 4(4): 45 - 49.

16. Silva A, Candida A, Lourenco M, Silva S. Evaluación de la calidad de los registros de enfermería en una unidad de cuidados semi intensivos. Rev. Esc. Anna Nery (Bra). 2010; 16 (3).

17. Mejía A, Dubón M, Carmona B, Ponce G. Nivel de apego de los registros clínicos de enfermería a la norma oficial Mexicana NOM - 168 - SSA1- 1998, del expediente clínico. Rev CONAMED. 2011; 16(1):4-10.

18. Anglade C. Características de las anotaciones de enfermería y factores personales e institucionales asociados a su elaboración en el hospital nacional "Dos de Mayo". Tesis de licenciatura. Lima, Perú: Universidad Nacional Mayor de San Marcos; 2006.

19. Gutierrez J, Esquén O, Gómez E. Nivel de cumplimiento de los registros de Enfermería en el servicio de Ginecoobstetricia del Hospital Nacional Madre Niño San Bartolomé 2012. Revista Científica de Ciencias de la Salud (Per). 2014; 7(1): 51-56. 